\title{
Hábitos alimenticios de Sphoeroides testudineus (Perciformes: Tetraodontidae) en el sistema lagunar de Ría Lagartos, Yucatán, México
}

\author{
Ariel Adriano Chi-Espínola \& María Eugenia Vega-Cendejas \\ Laboratorio de Taxonomía y Ecología de Peces, CINVESTAV-IPN, Unidad Mérida, km 6 antigua carretera a Progreso, \\ AP 73 Cordemex, C.P. 97310 Mérida, Yucatán, México; kain_ex@hotmail.com, maruvega@mda.cinvestav.mx
}

\author{
Recibido 29-II-2012. Corregido 10-IX-2012. Aceptado 05-X-2012.
}

\begin{abstract}
Feeding habits of Sphoeroides testudineus (Perciformes: Tetraodontidae) in the lagoon system of Ria Lagartos, Yucatán, Mexico. Sphoeroides testudineus is a dominant species in the coastal systems of Yucatán. Because of its wide distribution, occurrence and abundance performs an important functional role in coastal ecosystems. We assessed the trophic preferences and trophic-level variation in space and time for this species in Ria Lagartos lagoon, an hyperhaline ecosystem located Northwest of Yucatan Peninsula. The specimens were collected bimonthly during two annual periods (2004-2005 and 2007-2008) in 23 sites distributed along the system into four zones (marine, channel, Coloradas West and Coloradas East). Spatial and seasonal trophic variations were evaluated using canonical correspondence analysis (ACC). In a total of 382 individuals, 68 food resources included in 20 trophic groups were obtained. Higher relative importance index values (IIR) were obtained for bivalves, gastropods and macrophytes. Seasonal trophic variation showed that bivalves and gastropods were consumed along the year, while macrophytes were a preferential food during rains and windy seasons. Spatial variation indicates lower gastropods consumption at the inner zone of the system, and the opposite ocurred with bivalves. The consumption of macrophytes may be due to their greater abundance during rains and in the channel zone. The ACC showed that spatial trophic variation was due because of the abundance of the preferential preys (bivalves, gastropods), while seasonal differences by the secondary (amphipods, eggs, nematodes, brachyuran, detritus, nematodes). The results contribute to the biological knowledge of the species and highlight the importance and trophic function of $S$. testudineus for the energy transfer from the benthos to higher trophic levels of the food chain. Rev. Biol. Trop. 61 (2): 849-858. Epub 2013 June 01.
\end{abstract}

Key words: Sphoeroides testudineus, Ría Lagartos, feeding habits, bivalves, gastropods, macrophytes.

El conocimiento de la ictiología lagunar estuarina, es uno de los aspectos importantes en los estudios bio-ecológicos que pretenden evaluar y discutir los recursos bióticos presentes en un área costera (Mallard-Colmenero et al. 1982). Se han realizado investigaciones en función del conocimiento de estas áreas de alta productividad e importancia ecológica, por ser zonas de crianza y protección de larvas y juveniles que en su fase adulta forman parte de las pesquerías comerciales (Guevara et al. 2007).

Un aspecto de importancia entre las especies dominantes de los sistemas costeros es la transferencia de energía a través de los diversos componentes bióticos del sistema. En los ecosistemas costeros del sureste de México representantes de la familia Tetraodontidae son reconocidos por su amplia distribución, frecuencia y abundancia, considerándolos un recurso económico potencial de gran magnitud a nivel regional y nacional (Mallard-Colmenero et al. 1982). Adicionalmente, algunos de sus representantes son reconocidos por su valor como peces de ornato y en la industria farmacéutica por la tetrodotoxina presente en sus tejidos (Thomson et al. 2000, Ghosh et al. 2005, Moreno et al. 2009). Debido a estas características, se han realizado diversos estudios que contribuyen al conocimiento bio-ecológico de especies representativas, uno de estos 
son los análisis de contenidos estomacales, necesarios para entender y conocer su ecología trófica e importancia en la función de los ecosistemas costeros (Jackson et al. 2000, Moreno et al. 2009).

Es fundamental el conocimiento de las dietas, ecología de la alimentación y relaciones tróficas de los peces estuarinos al momento de tomar decisiones de manejo pesquero y conservación de los recursos. Dicha información relacionada con el requerimiento alimenticio, patrones de conducta alimentaria y las relaciones predador-presa, permite determinar el funcionamiento de la comunidad, así como interpretar o predecir cambios ocasionados por impactos de origen natural o de tipo antropogénico (Blaber 2000).

En particular sobre el ecosistema hiperhalino de la Reserva de la Biosfera Ría Lagartos, se han realizado diversos estudios sobre la estructura y uso de hábitat de la comunidad íctica (Vega-Cendejas \& Hernández de Santillana 2004, Peralta-Meixueiro \& Vega-Cendejas 2011). Sin embargo, no se ha abordado el aspecto de la biología trófica de las especies dominantes con el fin de evaluar su función en el ecosistema. Dentro de estas especies se encuentra Sphoeroides testudineus, reconocido en la región como botete (Nelson et al. 2004). En este sentido, el presente estudio tiene como objetivo determinar sus preferencias alimenticias y la variación trófica a nivel espacial y temporal en un ecosistema donde las condiciones de salinidad $(>40)$ pueden llegar a ser drásticas para la producción del sistema.

\section{MATERIALES Y MÉTODOS}

Área de estudio: La laguna de la Reserva de Ría Lagartos está comprendida entre los $21^{\circ} 26^{\prime}-21^{\circ} 38^{\prime}$ N y y $87^{\circ} 30^{\prime}-88^{\circ} 15^{\prime}$ W. Posee una extensión aproximada de $9371 \mathrm{ha}$, con un ancho que va de $25.0 \mathrm{~m}$ a $3.5 \mathrm{~km}$, longitud de $80 \mathrm{~km}$ y profundidad $0.5-3.0 \mathrm{~m}$. Se han reportado dos tipos de climas: BSo(h')w(x')iw" clima más seco de los áridos y Ax'(wo) iw" que corresponde al más seco de los cálidos húmedos, la temperatura media anual es de $22^{\circ} \mathrm{C}$. Existe una amplia diversidad de ambientes como manglar, selva mediana subperennifolia, selva baja caducifolia, vegetación de dunas costeras, petenes y sabana representada por tular, pastizal y carrizal (INE 1999).

Muestreo: Se realizaron muestreos bimensuales durante dos períodos anuales (2004-2005 y 2007-2008) que abarcaron las temporadas de lluvias (julio-octubre), nortes (noviembrefebrero) y secas (marzo-junio). Para la recolecta de los ejemplares se utilizó un chinchorro playero de $15.0 \mathrm{~m}$ de largo con $1.5 \mathrm{~m}$ de caída y $2.5 \mathrm{~cm}$ de abertura de malla, en 23 sitios distribuidos a lo largo del sistema agrupados en cuatro zonas (Marina, Canal, Coloradas oeste y Coloradas este) (Fig. 1). Estas localidades fueron establecidas previamente en función

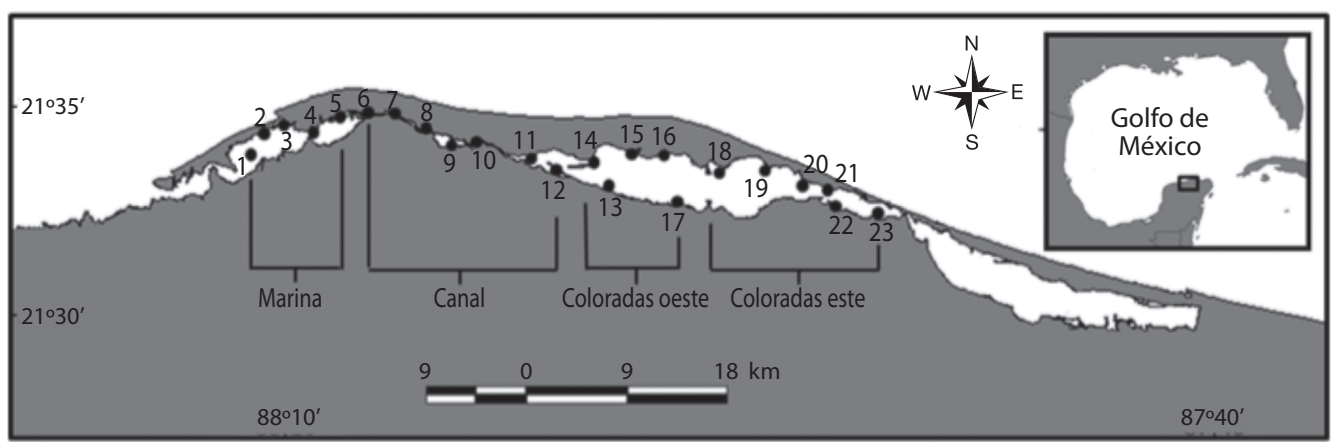

Fig. 1. Área de estudio y ubicación de los sitios y zonas de muestreo.

Fig. 1. Study area and location of sites and sampling areas. 
de la hidrología del sistema y tipos de hábitat (Peralta-Meixueiro \& Vega-Cendejas 2011). Se realizaron dos arrastres por sitio con una duración aproximada de 10min cada uno, entre las 8:00 y las 17:00h.

Los especímenes capturados fueron colocados en hielo y posteriormente inyectados con una solución de formol (10\%) para su preservación. En el laboratorio se enjuagaron con agua corriente y se preservaron con alcohol $(70 \%)$. Se registró de manera individual la longitud estándar en cm (LE) y el peso total (g). Del total de los especímenes recolectados, el 19\% se utilizó para el análisis trófico. Los componentes alimenticios se identificaron al nivel taxonómico más bajo posible según el grado de digestión, utilizando claves especializadas para cada grupo taxonómico (Morris 1973, Rehder 1981, Amos \& Amos 1985, Littler et al. 1989, Littler \& Littler 2000). Los componentes de la dieta se analizaron mediante los métodos de porcentaje de área (PA), que se refiere al área relativa de cada presa, que es una modificación del método volumétrico, utilizado cuando el contenido estomacal está constituido por presas muy pequeñas (ostrácodos, foraminíferos, diatomeas) y cuyo peso no es posible registrar en una balanza analítica (Vega-Cendejas 1990). La frecuencia de ocurrencia $(\mathrm{FO}=(\mathrm{n} /$ $\mathrm{NE}$ *(100)) que consiste en contabilizar las veces que aparece una presa determinada (n) en el contenido estomacal de un grupo de peces (NE) expresado en porcentaje (Tresierra \& Culquichicom 1995) y el índice de importancia relativa $(\mathrm{IIR}=(\% \mathrm{~N}+\% \mathrm{~W}) * \% \mathrm{FO})$ desarrollado por Pinkas et al. (1971) y modificado por Yáñez-Arancibia (1975a) e Hyslop (1980), que unifica los valores del porcentaje volumétrico $(\mathrm{N})$, gravimétrico (W) y frecuencia de ocurrencia (FO). El alimento se clasificó como preferencial ( $\mathrm{FO} \geq 50 \%$ ), secundario $(50 \% \geq \mathrm{FO} \geq 10 \%)$ y accidental $(\mathrm{FO} \leq 10 \%)$ (Yáñez-Arancibia 1975b), basado en la FO.

Para el análisis de la variación trófica espacial y temporal, se realizó un análisis de correspondencia canónica (ACC) (Ter Braak $\&$ Smilauer 2002), para las presas con las épocas climáticas (secas, lluvias y nortes) y zonas (marina, canal, Coloradas oeste, Coloradas este), incluyendo las tallas y peso como covariables. En ambos casos se realizó la prueba de Monte Carlo para determinar si las variables medidas poseen significancia en el estudio (Adjeroud et al. 1998, Kupschus \& Tremain 2001). Para ello se utilizó el paquete de estadística multivariada CANOCO 4.5 (Ter Braak \& Smilauer 2002).

\section{RESULTADOS}

Composición trófica: Se capturó un total de 2028 ejemplares de $S$. testudineus durante los dos periodos anuales, de los cuales se analizaron 387 individuos. En el periodo 20042005 se recolectaron 206 ejemplares y 181 en 2007-2008, con LE entre 1.5 a $18.5 \mathrm{~cm}$ y peso de 7.6 y $245.9 \mathrm{~g}$. Del total de los estómagos analizados, el $23.0 \%$ se encontró lleno, el $75.7 \%$ contenía algún tipo de alimento y el $1.3 \%$ no tenía alimento.

Se identificaron 68 tipos alimenticios, los cuales fueron agrupados en 20 categorías tróficas. El grupo de moluscos y macrófitas constituyeron los componentes alimenticios preferenciales. Dentro de los cuales, los bivalvos presentaron una FO de $68.3 \%$ y un IIR de $56.5 \%$; seguido por los gasterópodos y macrófitas con FO de 59.9 y $50.8 \%$ respectivamente, aunque con valores muy bajos de IIR (13.7 y $8.9 \%$ ). Las presas consideradas como secundarias estuvieron constituidas por los nemátodos, anfípodos, braquiuros, huevos, MONI (materia orgánica no identificada) y detritus. El resto de los componentes fueron considerados accidentales (FO $\leq 10 \%)$ (Cuadro 1).

Variación trófica espacio-temporal: Los componentes alimenticios de $S$. testudineus mostraron una variación temporal y espacial. Al eliminar del análisis a las especies incidentales o a las que presentaron un FO menor al $10 \%$, nuevamente se detectaron diferencias espacio-temporales en lo que respecta a la composición y abundancia de los recursos alimenticios. Para determinar los componentes alimenticios que originan estas diferencias espaciales y temporales, se decidió realizar una 
CUADRO 1

Espectro trófico del botete (S. testudineus) en el sistema lagunar de Ría Lagartos, Yucatán (n=382)

TABLE 1

Trophic spectrum of checkered puffer (S. testudineus) in lagoon system of Ria Lagartos, Yucatan ( $\mathrm{n}=382)$

\begin{tabular}{|c|c|c|c|c|c|c|c|c|c|}
\hline Grupo alimentario & $\% \mathrm{~N}$ & $\% \mathrm{~W}$ & FO & IIR & Grupo alimentario & $\% \mathrm{~N}$ & $\% \mathrm{~W}$ & FO & IIR \\
\hline Macrophyta & 11.50 & 6.09 & 50.78 & 8.96 & Decapoda & & & & \\
\hline Totales & 11.50 & 6.09 & & & Caridea & 0.01 & 0.01 & 0.52 & $0.6 \mathrm{E}-5$ \\
\hline Foraminifera & 0.14 & 0.03 & 6.55 & 0.01 & Anomura & & & & \\
\hline Totales & 0.14 & 0.03 & & & Hippoidea & 0.16 & 0.10 & 0.52 & 0.01 \\
\hline Mollusca & & & & & Paguroidea & 0.01 & 0.01 & 0.26 & $0.3 \mathrm{E}-5$ \\
\hline Gastropoda & 10.20 & 12.57 & 59.95 & 13.69 & Brachyura & 11.42 & 11.29 & 42.67 & 9.72 \\
\hline Bivalvia & 37.12 & 45.34 & 68.33 & 56.52 & Totales & 11.60 & 11.40 & & \\
\hline Totales & 47.31 & 57.91 & & & Peces & & & & \\
\hline Nematoda & 0.07 & 0.01 & 10.47 & 0.01 & Osteichthyes & 0.30 & 0.29 & 3.14 & 0.02 \\
\hline Totales & 0.07 & 0.01 & & & Totales & 0.30 & 0.29 & & \\
\hline Annelida & & & & & Huevos & 1.09 & 0.91 & 12.04 & 0.24 \\
\hline Polychaeta & 1.17 & 1.35 & 9.42 & 0.24 & Totales & 1.09 & 0.91 & & \\
\hline Totales & 1.17 & 1.35 & & & MONI & 5.02 & 3.69 & 25.13 & 2.20 \\
\hline Crustacea & & & & & Totales & 5.02 & 3.69 & & \\
\hline Thecostraca & 4.31 & 5.74 & 8.90 & 0.90 & Detritus & 12.68 & 9.99 & 26.18 & 5.95 \\
\hline Ostracoda & 0.04 & 0.01 & 4.71 & 0.01 & Totales & 12.68 & 9.99 & & \\
\hline Malacostraca & & & & & Otros & 0.14 & 0.06 & 1.31 & 0.01 \\
\hline Amphipoda & 3.84 & 1.87 & 24.35 & 1.39 & Totales & 0.14 & 0.06 & & \\
\hline Isopoda & 0.66 & 0.62 & 9.42 & 0.12 & & & & & \\
\hline Tanaidacea & 0.14 & 0.03 & 8.12 & 0.01 & & & & & \\
\hline Totales & 8.99 & 8.27 & & & & & & & \\
\hline
\end{tabular}

*Donde: $\% \mathrm{~N}=$ Abundancia relativa, $\% \mathrm{~W}=\mathrm{Peso}, \mathrm{FO}=$ frecuencia de ocurrencia, $\mathrm{IIR}=$ Índice de importancia relativa.

serie de ACC mediante combinaciones con las presas preferenciales o secundarias contra las variables zonas o temporadas (Cuadro 2).

Se obtuvo que la abundancia de las presas secundarias es igual entre zonas, mientras que los recursos preferenciales son los que generan esta diferencia en la abundancia (ACC, $\mathrm{p}<0.05$ ) (Fig. 2A). Con respecto a la temporalidad, se encontró que $S$. testudineus consume las presas preferenciales en abundancias similares. Sin embargo, se registraron diferencias en lo que respecta a los recursos secundarios (ACC, $\mathrm{p}<0.05$ ) (Fig. 2B).

\section{DISCUSIÓN}

Composición trófica: En el presente estudio se determinaron un total de 68 tipos de presas que fueron incluidos en 20 categorías tróficas. Targett (1978) y Palacios-Sánchez \& Vega-Cendejas (2010) reportaron un número mayor de grupos tróficos para esta especie, mientras que Mallard-Colmenero et al. (1982), Valdivia (1996), Vasconcelos-Filho et al. (1998), Vega-Cendejas (1998), Guevara et al. (2007), Chiaverini (2008) y Barros et al. (2010) obtuvieron un número menor. Sobre este aspecto, hay que resaltar que la composición y abundancia de los recursos alimenticios presentan variaciones entre sitios y temporalmente en función de la disponibilidad y ocurrencia de las presas, así como la intensidad en el esfuerzo de muestreo. Este trabajo incorpora información que se desconocía en cuanto a los hábitos alimenticios de S. testudineus en la Península de Yucatán, y aun más en la 
CUADRO 2

Valores generados por el ACC con las variables temporales y espaciales, los valores de F y P son generados por la prueba de Monte Carlo

TABLE 2

Values generated by the ACC with temporal and spatial variables, the values of F and P are generated by Monte Carlo test

\begin{tabular}{lccccccc}
\multicolumn{1}{c}{ Variable } & Grupo alimenticio & Primer eje & Segundo eje & Inercia & Varianza acumulada & F & P \\
Temporada & $\mathrm{P}, \mathrm{S}, \mathrm{I}$ & 82.7 & 100 & 7.405 & 1.603 & 3.007 & $0.002^{*}$ \\
Zona & $\mathrm{P}, \mathrm{S}, \mathrm{I}$ & 66.2 & 94.7 & 7.405 & 5.034 & 6.098 & $0.002^{*}$ \\
Temp/Zona & $\mathrm{P}, \mathrm{S}$ & 83.7 & 91.0 & 3.771 & 1.535 & 8.879 & $0.002^{*}$ \\
Temporadas & $\mathrm{P}, \mathrm{S}$ & 69.5 & 100 & 3.771 & 0.192 & 2.910 & $0.006^{*}$ \\
& $\mathrm{P}$ & 81.6 & 100 & 1.455 & 3.403 & 0.258 & 0.916 \\
& $\mathrm{~S}$ & 74.4 & 100 & 3.127 & 8.705 & 5.361 & $0.002^{*}$ \\
Zonas & $\mathrm{P}, \mathrm{S}$ & 83.8 & 98 & 3.771 & 2.079 & 11.856 & $0.002^{*}$ \\
& $\mathrm{P}$ & 50.3 & 100 & 1.455 & 3.268 & 29.710 & $0.002 *$ \\
& $\mathrm{~S}$ & 61.4 & 81.3 & 3.771 & 10.637 & 8.879 & 0.010 \\
\hline
\end{tabular}

Grupo alimenticio: $\mathrm{P}=$ preferenciales, $\mathrm{S}=$ secundarios, $\mathrm{I}=$ incidentales. ${ }^{*} \mathrm{p}<0.05$

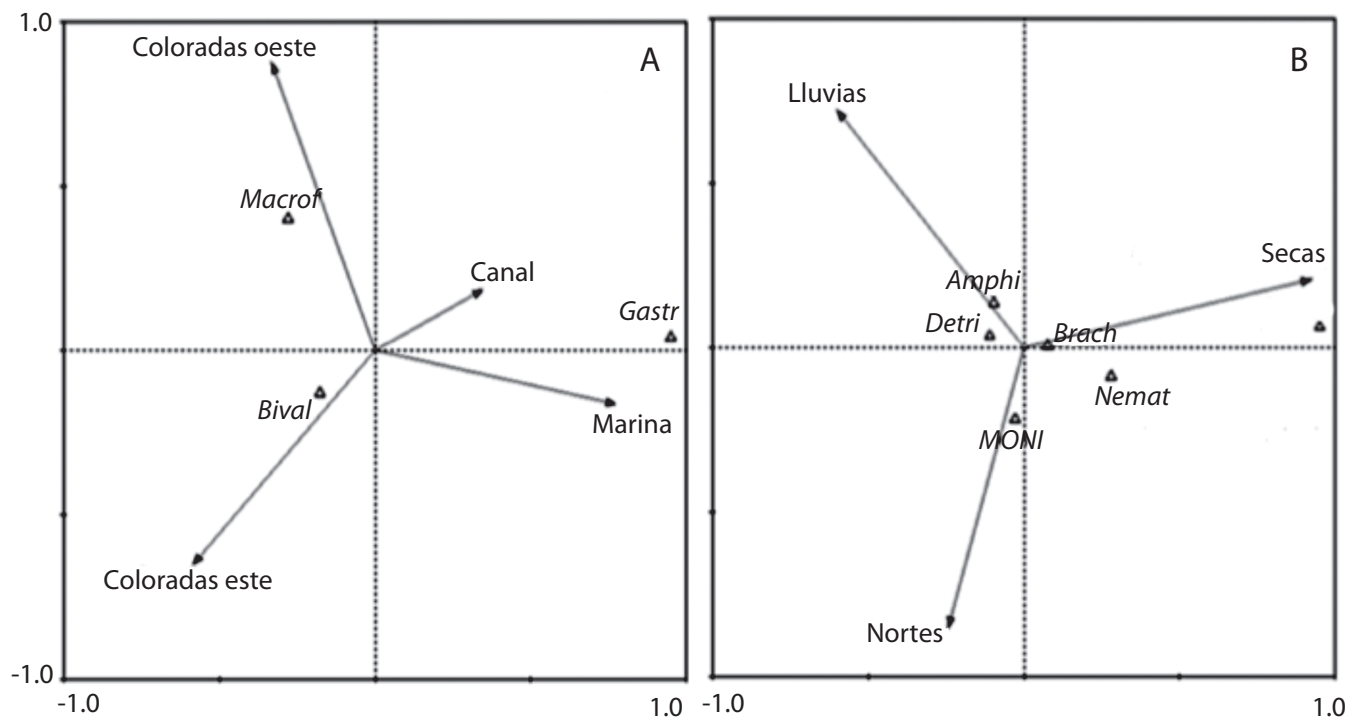

Fig. 2. Biplot del ordenamiento de ACC de la proporción de los recursos alimenticios del botete (S. testudineus). (A) variables espaciales y presas principales. (B) variables temporales y presas secundarias.

Fig. 2. ACC biplot of the proportion of the food resources of the checkered puffer (S. testudineus). (A) spatial variables and major preys, (B) temporary variables and secondary preys.

Laguna de Ría Lagartos. Estudios previos en la bocana de la carbonera (Palacios-Sánchez \& Vega-Cendejas 2010), señalan a esta especie junto con $S$. spengleri como un depredador de organismos bentónicos, con una marcada preferencia hacia los bivalvos y con una repartición de las zonas de alimentación en relación con la ocurrencia, abundancia y talla de las especies.

La preferencia alimentaria de S. testudineus está dominada por los grupos de bivalvos, gasterópodos y macrófitas. Estos resultados concuerdan con lo reportado por Targett (1978), 
Mallard-Colmenero et al. (1982), Vega-Cendejas (1998), Chiaverini (2008) y PalaciosSánchez \& Vega-Cendejas (2010), los cuales encontraron altas frecuencias de gasterópodos y bivalvos en la dieta de esta especie, aunque algunos de ellos reportan a los crustáceos (braquiuros) como un elemento frecuente, mismo que en este trabajo quedó como un alimento secundario para la zona de Ría Lagartos.

Entre las características intrínsecas de la especie, que son determinantes para encontrar ciertos elementos alimenticios como representativos en su espectro trófico, está el tipo de dientes en forma de placas que les permite triturar las conchas de los moluscos y caparazones quitinosos de los crustáceos, además de su proximidad con la infauna del sedimento por sus hábitos bénticos (Vega-Cendejas 1998).

La presencia de las macrófitas (Halodule) como preferencial en la dieta de S. testudineus, de igual manera pudo estar asociado con la voracidad de esta especie al capturar sus presas entre las estructuras de las plantas acuáticas, lo que puede implicar un consumo accidental (Chiaverini, 2008), puesto que algunos estudios hacen énfasis en su carnivoría (Targett 1978, Keith et al. 2000). Otro aspecto que hay considerar es la mayor abundancia de este componente alimenticio en la época de lluvias y en la zona del canal y parte de Coloradas oeste, donde la salinidad decrece considerablemente (20.7) (Peralta-Meixueiro \& Vega-Cendejas 2011).

En cuanto al IIR, los valores más altos se registraron en relación al consumo de bivalvos, gasterópodos, braquiuros y macrófitas. Estos resultados coinciden con lo establecido con anterioridad en el trabajo de Mallard-Colmenero et al. (1982) y Guevara et al. (2007). No obstante, ambos estudios resaltan a MONI de importancia como un componente alimenticio, lo que difiere a los resultados de este estudio. En este sentido, cabe resaltar que la cantidad de MONI va a depender del grado de digestión o conservación de los recursos alimenticios.

La ingestión de ciertos elementos como los insectos, peces, corales y ofiúridos, parece haber sido accidental, ya que fueron ocasionales. Es muy probable que estas presas estuvieran sobre el sustrato y se ingirieran accidentalmente. Adicionalmente la cantidad de sedimentos en el contenido alimenticio de esta especie, se puede atribuir al hábito de alimentarse cerca del sustrato (Robins \& Ray 1986, Chiaverini 2008).

Variación trófica espacio-temporal: El conocimiento del uso espacial y temporal del hábitat permite inferir sobre las relaciones ecológicas entre organismos, esto se refleja en la composición de sus dietas, que permite establecer diferentes niveles tróficos y la evaluación de un hábitat determinado (Sánchez et al. 1996). La adquisición de conocimientos de los hábitos alimenticios de las especies facilita entender la forma en que se desarrollan sus ciclos de vida (Wootton 1990, Guevara et al. 2007).

De acuerdo al análisis espacial, se observa que el valor de IIR de los gasterópodos, se reduce hacia el interior de la laguna mientras que lo contrario sucede con los bivalvos, con un incremento del IIR hacia su interior (Fig. 3A). Esta variación trófica se puede deber a que el bivalvo del género Anomalocardia es muy tolerante a las altas salinidades (Silva et al. 2005), las cuales se registraron en la zona del Coloradas este $(>60)$. Por otra parte en la zona de canal y Coloradas oeste, donde la salinidad decrece hasta 35-50 la presencia de macrófitas como sustrato puede ser el factor que generó su presencia en el contenido estomacal (PeraltaMeixueiro \& Vega-Cendejas 2011).

Los recursos alimenticios registrados en todas las épocas climáticas fueron siempre los bivalvos y gasterópodos (Fig. 3B); coincidiendo con lo observado por Mallard-Colmenero et al. (1982), Valdivia (1996), Chiaverini (2008) y Palacios-Sánchez \& Vega-Cendejas (2010), que establecen que el tipo de alimentación de $S$. testudineus está constituido por crustáceos y moluscos. Únicamente se registraron a las macrófitas en la categoría de preferenciales durante las épocas de lluvias y nortes, donde es muy probable que el incremento de la precipitación y consecuentemente de la materia orgánica, así como la disminución de la salinidad 

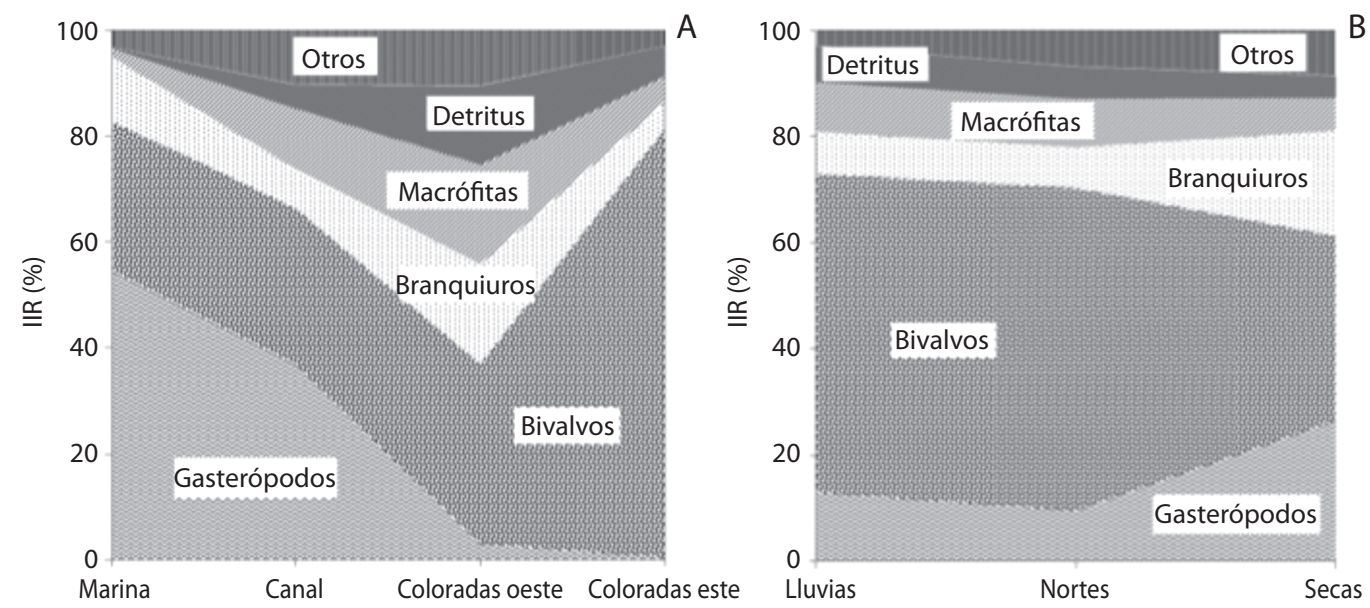

Fig. 3. Principales recursos alimenticios del botete (S. testudineus) durante los dos periodos de muestreo con base en su (A) variación espacial y (B) temporal.

Fig. 3. Main food sources of the checkered puffer (S. testudineus) during the two sampling periods based on their (A) spatial and (B) temporal.

en el sistema, producen un incremento de su biomasa y consecuentemente de la abundancia de los recursos alimenticios disponibles (Kolm \& Andretta 2003).

La composición y abundancia de los recursos alimenticios mostró diferencias significativas entre las variables tanto espacial como temporalmente. Con el fin de evitar un sesgo debido a la tendencia alimentaria hacia un tipo de talla o peso del pez, la longitud patrón y peso fueron incluidos en el ACC como covariables. Las diferencias espaciales son generadas por las proporciones de las presas preferenciales (bivalvos, gasterópodos y macrófitas), mientras que las diferencias temporales son originadas por las presas secundarias (braquiuros, anfípodos, detritus, nemátodos, MONI y huevos). Aunque Mallard-Colmenero et al. (1982) no encontraron diferencias entre las presas tanto a nivel espacial como temporal, Chiaverini (2008) registró diferencias temporales, donde S. testudineus presentó mayor actividad de alimentación en invierno (nortes). No obstante estos resultados discrepan con lo obtenido, cabe mencionar que es el primer estudio de alimentación en $S$. testudineus realizado en esta laguna, donde existen condiciones bióticas y abióticas distintas a otras lagunas.
La proporción de presas preferenciales con la variable espacial (marina, canal, Coloradas este y Coloradas oeste), muestra que los bivalvos y macrófitas están correlacionadas a las zonas de Coloradas este y oeste, mientras que los gasterópodos están mayormente correlacionados con la zona marina y canal. Al respecto, Peralta-Meixueiro \& Vega-Cendejas (2011) establecen para el mismo sitio, una alta abundancia de Halodule wrightii (macrófita) en las zonas de canal, Coloradas este y oeste, además de la ausencia de T. testudinum, debido posiblemente al paso del huracán Isidoro dado que $H$. wrightii es una especie oportunista (Herrera-Silveira et al. 2000).

Considerando la proporción de presas secundarias con la variable temporal, se muestra una abundancia similar entre épocas, excepto por la presencia y proporción de huevos que se encontraron muy correlacionados con la temporada de secas. Al respecto, Chiaverini (2008) comenta que durante la primavera (secas), es la estación más favorable para la reproducción de varias especies. También es la época en que inician las precipitaciones y por consiguiente, el incremento de materia orgánica en circulación (Kolm \& Andretta 2003), e incremento de los principales componentes 
alimenticios de varias especies (Castro et al. 2004). Con estos resultados podemos establecer que S. testudineus es un depredador específico, cuyas principales presas corresponden a bivalvos y gasterópodos. Asimismo se observa una variación espacial y temporal del uso de los recursos alimenticios, en función de su abundancia y en donde la salinidad es el parámetro que limita su distribución a lo largo del sistema.

\section{AGRADECIMIENTOS}

A Mirella Hernández de Santillana por su valiosa ayuda en la recolecta e identificación de las muestras, a Jorge Montero Muñoz por sus sugerencias y apoyo en los análisis estadísticos, al grupo de trabajo del laboratorio de taxonomía y ecología de peces. Finalmente externar nuestro agradecimiento al grupo anónimo de revisores por sus comentarios y sugerencias al documento.

\section{RESUMEN}

Sphoeroides testudineus, es una especie dominante en los sistemas costeros de Yucatán. Debido a su amplia distribución, ocurrencia y abundancia realiza un importante papel funcional en los ecosistemas costeros. Evaluamos para esta especie las preferencias tróficas y su variación a nivel espacial y temporal en la laguna de Ría Lagartos, un ecosistema hiperhalino localizado al noroeste de la península de Yucatán. Los especímenes fueron recolectados bimensualmente durante dos periodos anuales (2004-2005 y 2007-2008) en 23 sitios distribuidos a lo largo del sistema en cuatro zonas (marina, canal, Coloradas oeste y Coloradas este). Las variaciones tróficas espaciales y temporales fueron evaluadas mediante un análisis de correspondencia canónica (ACC). En un total de 382 individuos, se obtuvieron 68 recursos alimenticios incluidos en 20 grupos tróficos. El mayor índice de importancia relativa (IIR) 1o obtuvieron los bivalvos, gasterópodos y macrófitas. La variación trófica estacional mostró que los bivalvos y gasterópodos son consumidos a lo largo del año, mientras que las macrófitas constituyeron el alimento preferencial en la temporada de lluvias y nortes. La variación espacial indica un bajo consumo de gasterópodos hacia el interior de la laguna, y lo opuesto sucedió con los bivalvos. El consumo de macrófitas pudo ser debido a su mayor abundancia durante lluvias y en la zona del canal. El ACC mostró que la variación trófica espacial se debió a la abundancia de las presas preferenciales (bivalvos y gasterópodos), mientras que las diferencias estacionales por las presas secundarias (anfípodos, huevos, nematodos, braquiuros, detritus, nemátodos). Los resultados contribuyen al conocimiento biológico de la especie y resaltan la importancia y función trófica de Sphoeroides testudineus en las transferencias de energía desde el bentos hacia los niveles tróficos superiores de la cadena alimenticia.

Palabras clave: Sphoeroides testudineus, Ría Lagartos, hábitos alimenticios, bivalvos, gasterópodos, macrófitas.

\section{REFERENCIAS}

Adjeroud, M., Y. Letourneur, M. Porcher \& B. Salvat. 1998. Factors influencing spatial distribution of fish on a fringing reef at Mauritius, S.W. Indian Ocean. Environ. Biol. Fish. 53: 169-182.

Amos, W. \& S. Amos. 1985. Atlantic \& Gulf Coasts. The Audubon Society Nature Guides, Nueva York, EEUU.

Barros, J., J. Francisco, A. Vasconcelos-Filho \& D. Tenorio. 2010. Moluscos encontrados no trato digestivo de Sphoeroides testudineus (Linnaeus, 1758) (Teleostei: Tetraodontidae), no Canal de Santa Cruz, ItamaracáPernambuco, Brasil. Rev. Bras. Enga. Pesca 5: 70-80.

Blaber, S. 2000. Tropical Estuarine Fishes: Ecology, Exploitation and Conservation, Blackwell Science, Londres, Inglaterra.

Castro, L., A. Acero \& A. Santos-Martínez. 2004. Ecología trófica de la Carrura Bairdiella ronchus (Pisces: Sciaenidae) en la Ciénaga Grande de Santa Marta, Caribe Colombiano. Rev. Acad. Colomb. Cienc. 28: 529-534.

Chiaverini, A. 2008. Ecología trófica de Sphoeroides testudineus (Linnaeus 1758). Sphoeroides greeleyi (Gilbert 1900) Da Gamboa Do Perequê, Pontal Do Sul, Paraná, Brasil. Tesis de Maestría, Universidad Federal de Paraná, Paraná, Brasil.

Ghosh, S., A. Hazra, S. Banerjee \& B. Mukherjee 2005. Ecological monitoring for ascertaining the bio-safety of liver lipids from some Indian marine puffer fishes. Fish. Sci. 71: 29-37.

Guevara, E., A. Sánchez, C. Rosas, M. Mascaró \& R. Brito. 2007. Asociación trófica de peces distribuidos en vegetación acuática sumergida en Laguna de Términos, sur del Golfo de México. U. Cienc. Tróp. Húm. 23: 151-166.

Herrera-Silveira, J., J. Ramírez-Ramírez, N. Gómez \& A. Zaldivar. 2000. Seagrass bed recovery after hydrological restoration in a coastal lagoon with groundwater discharges in the north of Yucatán, p. 123-135. In S. Bortone (ed.). Seagrasses: Monitoring, Ecology, Physiology and management. Blackwell, Londres, Inglaterra. 
Hyslop, E. 1980. Stomach contents analysis, a review of methods and their application. J. Fish Biol. 17: 411-429.

INE. 1999. Programa de manejo de la Reserva de la Biosfera de Ría Lagartos. Yucatán, México.

Jackson, G., N. Buxton \& M. George. 2000. Diet of the southern opah Lampris immaculatus on the Patagonoian Shelf; significance of the squid Morotheuthis ingens and anthropogenic plastic. Mar. Ecol. Prog. Ser. 206: 261-271.

Keith, P., Y. Le Bail \& P. Planquette. 2000. Atlas des poissons d'eau douce de Guyane (tome 2, fascicule I). Publications scientifiques du Muséum, Paris, Francia.

Kolm, H. \& L. Andretta. 2003. Bacterioplancton in different tides of the Pereque tidal creek, Pontal do Sul, Paraná, Brasil. Braz. J. Microbiol. 34: 97-103.

Kupschus, S. \& D. Tremain. 2001. Associations between fish assemblages and environmental factors in nearshore habitats of a subtropical estuary. J. Fish Biol. 5: $1383-1403$.

Littler, D. \& M. Littler. 2000. Caribbean Reef Plants, An identification Guide to the Reef Plants of the Caribbean, Bahamas, Florida, and Gulf of México. Offshore Graphics, Washington D.C., EEUU.

Littler, D., M. Littler, K. Bucher \& J. Norris. 1989. Marine Plants of the Caribbean, a field guide from Florida to Brazil. Smithsonian Institution, Washington D.C., EEUU.

Mallard-Colmenero, L., A. Yáñez-Arancibia \& F. Amezcua-Linares. 1982. Taxonomía, diversidad, distribución y abundancia de los tetraodontidos de la Laguna de Términos, sur del Golfo de México. (Pisces: Tetraodontidae). An. Inst. Cienc. del Mar y Limnol. Univ. Nal. Autón. Mex. 9: 161-212.

Moreno, X., A. Abitia, A. Favila, F. Gutiérrez \& D. Palacios. 2009. Ecología trófica del pez Arothron meleagris (Tetraodontiformes: Tetraodontidae) en el arrecife de Los Frailes, Baja California Sur, México. Rev. Biol. Trop. 57: 113-123.

Morris, P. 1973. A Field Guide to Shells of the Atlantic and Gulf Coasts and the West Indies. The Peterson Field Guide Series, Boston, EEUU.

Nelson, J., E. Crossman, H. Espinosa-Pérez, L. Findley, C. Gilbert, R. Lea \& J. Williams. 2004. Common and scientific names of fishes from the United States, Canada, and Mexico. American Fisheries Society, Special Publication 29, Bethesda, Maryland, EEUU.

Palacios-Sánchez, S. \& M.E. Vega-Cendejas. 2010. Cambios alimenticios en tres especies de Sphoeroides (Tetraodontiformes: Tetraodontidae) posterior al huracán Isidoro en Bocana de la Carbonera, Sureste del Golfo de México. Rev. Biol. Trop. 58: 1223-1235.

Peralta-Meixueiro, M. \& M.E. Vega-Cendejas. 2011. Spatial and temporal structure of fish assemblages in a hyperhaline coastal system: Ría Lagartos, Mexico. Neotrop. Ichthyol. 9: 673-682.

Pinkas, L., M. Olipant \& Z. Iverson. 1971. Food habits of albacore bluefin, tuna and bonito in California Waters. Fish. B. 152: 1-1105.

Rehder, H. 1981. National Audubon Society Field Guide to North American Seashells. Alfred a Knopf, Nueva York, EEUU.

Robins, C. \& G. Ray. 1986. A field guide to Atlantic coast fishes of North America. Houghton Mifflin Company, Boston, EEUU.

Sánchez, A., A. Raz-Guzman \& E. Barba. 1996. Habitat value of seagrasses for decapods in tropical coastal lagoons of the south western Gulf of Mexico: An overview, p. 233-240. In J. Kuo, C. Phillips, D. Walker \& H. Kirkman (eds.). Seagrass Biology. Proceedings of an International Workshop. Rottnest Island, Australia.

Silva, E., A. Soares-Gomes, F. Fernandes \& C. Abreu. 2005. Sandy beach macrobenthos assemblages at a hypersaline coastal lagoon, Lagoa de Araruama, RJ, Brasil. J. Coast. Res. 42: 265-270.

Targett, T. 1978. Food resource partitioning by the pufferfishes Sphoeroides spengleri and Sphoeroides testudineus from Biscayne Bay. Florida. Mar. Biol. 49: 83-91.

Ter Braak, C. \& P. Smilauer. 2002. CANOCO Reference Manual and CanoDraw for Windows User's Guide: Software for Canonical Community Ordination (version 4.5). Microcomputer Power,Nueva York, EEUU.

Thomson, D., L. Findley \& A. Kerstitch. 2000. Reef fishes of the Sea of Cortez. University of Texas, Austin, Texas, EEUU.

Tresierra, A. \& G. Culquichicom. 1995. Manual de Biología Pesquera. Trujillo, Distrito Federal, México.

Valdivia, F. 1996. Aspectos de la biología de Sphoeroides testudineus \& S. nephelus, en la laguna de Tamiahua, Veracruz, México. Tesis de Licenciatura, Universidad Nacional Autónoma de México, Distrito Federal, México.

Vasconcelos-Filho, A., K. Silva \& F. Acioli. 1998. Hábitos alimentares de Sphoeroides testudineus (Linnaeus, 1758) (Teleostei: Tetraodontidae), no canal de Santa Cruz, Itamaracá-PE. Trab. Oceanogr. Univ. Feb. Pernambuco 26: 145-157.

Vega-Cendejas, M.E. 1990. Interacción trófica entre los bagres Arius melanopus (Agassiz, 1929) y Arius felis (Linnaeus, 1766) en las costas de Celestún, Yucatán, México. An. Inst. Cienc. del Mar y Limnol. Univ. Nal. Autón. Mex. 17: 271-285.

Vega-Cendejas, M.E. 1998. Trama trófica de la comunidad nectónica asociada al ecosistema de manglar en el litoral norte de Yucatán. Tesis de Doctorado, Universidad Nacional Autónoma de México, Distrito Federal, México. 
Vega-Cendejas, M.E. \& S.M. Hernández de Santillana. 2004. Fish community structure and dynamics in a coastal hypersaline lagoon: Rio Lagartos, Yucatán, México. Est. Coast. Shelf Sci. 60: 285-299.

Wootton, R. 1990. Ecology of teleost fishes. Fish and Fisheries series 1. Chapman and Hall, Londres, Inglaterra.

Yáñez-Arancibia, A. 1975a. Informe Final de 2da. Etapa del Programa de Uso de la Zona Costera de Michoacán y
Guerrero. Relaciones tróficas de la fauna ictiológica del sistema lagunar costero de Guerrero y aspectos parciales de dinámica de poblaciones de los peces de importancia comercial. Convenio Comisión del Río Balsas, S. R. H. y Centro de Ciencias del Mar y Limnología, U. N. A. M., México. Contrato N9 OC-E-03-74. $750 \mathrm{p}$.

Yáñez-Arancibia, A. 1975b. Estudios de peces en las lagunas costeras: nota científica. An. Centro Cienc. del Mar y Limnol. Univ. Nal. Autón. 2: 53-60. 